\title{
ARGILAS PILARIZADAS COM POLIHIDROXICATIONS DE ALUMÍNIO: OTIMIZAÇÃO DO USO DA ÁGUA NO PROCESSO
}

\author{
Pillarized clays with aluminum polyhydroxications: optimization of the use of \\ water in the process
}

Damiana Sinezio de Souza ${ }^{1,2}$; Fabio Garcia Penha ${ }^{2}$; Sibele Berenice Castellã Pergher ${ }^{1 *}$

${ }^{1}$ LABPEMOL - Laboratório de Peneiras Moleculares, Instituto de Química, Universidade Federal do Rio Grande do Norte. Campus Universitário, Lagoa Nova, Natal, RN. *e-mail: sibele.pergher@ufrn.br

${ }^{2}$ Instituto Federal do Rio Grande do Norte. Nova Cruz - RN.

Data do recebimento: 18/07/2021 - Data do aceite: 13/08/2021

RESUMO: Uma argila comercial (Aldrich), predominantemente Montmorillonita, foi pilarizada com polihidroxications de alumínio (tipo ion de Keggin). No processo de pilarização foi estudada a otimização do uso da água em três etapas diferentes: na primeira o uso ou não de água para pré-expansão da argila; numa segunda etapa se estudou a quantidade de solução do agente pilarizante a ser empregada variando de $100 \mathrm{~mL} / \mathrm{g}$ a $300 \mathrm{~mL} / \mathrm{g}$; finalmente, na terceira etapa, se avaliou a lavagem do sólido obtido até teste negativo para cloretos. Os materiais obtidos foram caracterizados por difração de raios X. Os resultados apontaram que a etapa de pré-expansão da argila conduz a materiais melhores pilarizados e com espaçamentos basais maiores, mas pode ser reduzida ou até mesmo eliminada. A quantidade de solução de agente pilarizante de $100 \mathrm{~mL} / \mathrm{g}$ se mostrou ideal para obtenção de materiais pilarizados de forma ordenada, sendo uma quantidade muito menor da normalmente empregada $(250 \mathrm{~mL} / \mathrm{g})$. A lavagem demonstrou ser uma etapa fundamental para que os materiais após a calcinação se mantenham pilarizados.

Palavras-chave: Argilas Pilarizadas. Otimização de síntese. Íon de Keggin. Montmorillonita. 
ABSTRACT: Commercial clay (Aldrich) predominantly Montmorillonite was pillared with aluminum polyhydroxycations (Keggin ion type). In the pillaring process, the optimization of water use in three different stages was studied: first step, the use or not of water for the pre-expansion of the clay; second step, the amount of pillaring agent solution to be used, ranging from $100 \mathrm{~mL} / \mathrm{g}$ to $300 \mathrm{~mL} / \mathrm{g}$. Finally, in the third step, the washing of the solid obtained until negative test for chlorides was evaluated. The materials obtained were characterized by X-ray diffraction. The results showed that the clay pre-expansion step leads to better pillared materials and with larger basal spacing, but it can be reduced or even eliminated. The amount of pillaring agent solution of $100 \mathrm{~mL} / \mathrm{g}$ proved to be ideal for obtaining pillared materials in an orderly manner, with a much smaller amount than that normally used $(250 \mathrm{~mL} / \mathrm{g})$. Washing proved to be a fundamental step for the materials to remain pillared after calcination.

Keywords: Pillarized clays. Synthesis optimization. Keggin ion, Montmorillonite.

\section{Introdução}

Argilas são materiais naturais que englobam uma variedade de argilominerais com estruturas e composições diferentes (GUGGENHEIM et al., 2006). Dentre estes argilominerais se destaca a Montmorillonita que é um aluminossilicato lamelar pertencente ao grupo das esmectitas (ODOM, 1994). A montmorillonita tem sido muito empregada em processos de adsorção e catálise devido às suas propriedades de troca catiônica e acidez (PERGHER; SPRUNG, 2005; COGO, 2011). Sua estrutura lamelar é formada pela união de folhas formadas por tetraedros de $\mathrm{SiO}_{4}$ unidos a uma folha central de octaedros de $\mathrm{AlO}_{6}$, formando a conhecida camada 2:1 (PERGHER, 1993). (Figura 1)

Estas camadas estão carregadas negativamente devido a substituições isomórficas de Si por Al, que faz com que existam cátions interlamelares para compensação desta carga. Isto confere a estes materiais uma capacidade de troca catiônica que é responsável pelo seu emprego em diversos processos (PERGHER, 1994).

As camadas 2:1 da montmorillonita normalmente se encontram empilhadas devido a forças de Wan der Walls, e em sua região interlamelar (entre as camadas) existem os cátions compensadores de carga. Estes cátions se hidratam ao entrar em contato com a água, ocorrendo a expansão das camadas (aumento da distância entre camadas, aumento da região interlamelar) e são suscetíveis a troca. Esta estrutura particular deste tipo de argilomineral faz com que possuam alta capacidade de troca iônica, elevada área específica, capacidade de expansão que chega a ser de 20 vezes maior que o tamanho original, propriedade de intercalação (inserir compostos na região interlamelar), resistência à temperatura e aos solventes (AZEEZ et al., 2013; CARDONA et al., 2021).

A soma da espessura da camada 2:1 $(0,96 \mathrm{~nm})$ e da região interlamelar é chamada de espaçamento basal (d001) e é determinada pela difração de raios X. A quantidade total de cátions na região interlamelar do argi- 
Figura 1 - Estrutura do Argilomineral Montmorillonita. À esquerda se observa as unidades de tetraedros de $\mathrm{SiO}_{4}$ que se unem formando uma folha contínua (folha tetraédrica) e as unidades de octaedros de $\mathrm{AlO}_{6}$ que se unem formando uma folha continua (folha octaédrica). Estas folhas se conectam formando a camada 2:1 (a direita) constituída de suas folhas tetraédricas e uma central octaédrica. Entre as camadas se têm cátions de compensação de carga $\mathrm{M}^{\mathrm{n}+}$ que estão hidratados

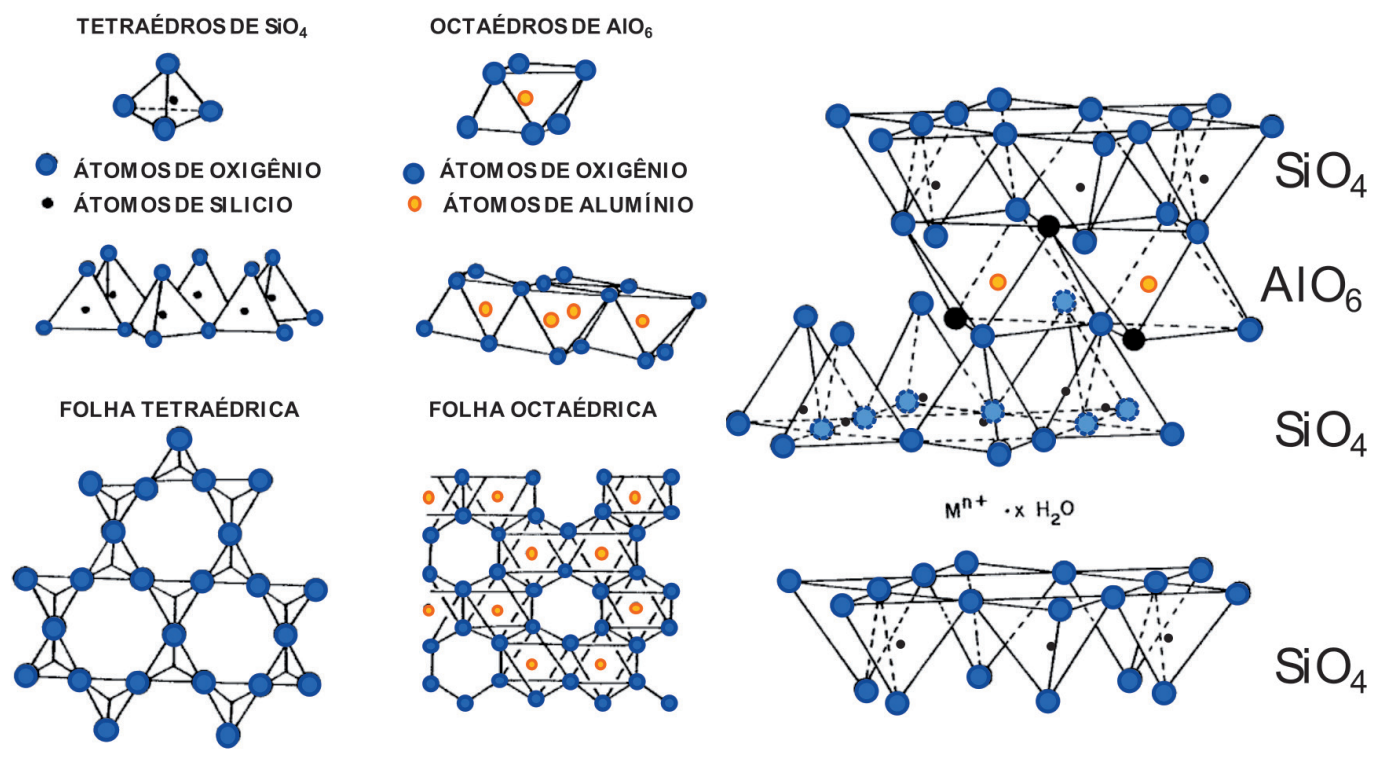

Fonte: Adaptada de PERGHER, 1994.

lomineral se refere à capacidade de troca catiônica (CTC), que é medida em unidade de $\mathrm{mmol} / 100 \mathrm{~g}$ e as montmorillonitas, normalmente, apresentam um valor de 150 mmol/100g (ROMANZINI, 2016).

A espessura da camada de água interlamelar varia com a natureza do cátion adsorvido e da quantidade de água disponível. Quando o cátion é o sódio, o inchamento pode progredir desde $0,98 \mathrm{~nm}$, quando a argila é exposta ao ar, a um máximo de $4,0 \mathrm{~nm}$, quando a argila é totalmente dispersa em meio líquido (AZZEZ et al., 2013).

Uma vez que as camadas estão empilhadas umas sobre as outras, a superfície acessível para adsorção de moléculas, acaba sendo a superfície externa dessas camadas empilhadas. Algumas moléculas, se pequenas ou suficientemente polares, podem ingressar na região interlamelar. Para aumentar o acesso de moléculas (tanto para adsorção como para catálise) a superfície destes argilominerais, existem alguns tratamentos como desorganização das camadas (desempilhamento ou esfoliação), separação das camadas pela inserção de compostos que funcionarão como pilares, entre outros (WEN et al., 2019).

A este último processo, se chama como Pilarização de Argilas, que consiste em três etapas: i) tratamento com água para a expansão das camadas através da hidratação dos cátions interlamelares; ii) troca catiônica dos cátios interlamelares $\left(\mathrm{Ca}^{+2}, \mathrm{Na}^{+}\right.$, etc) por cátions maiores que funcionarão como separadores das camadas, pilarização propriamente dita e, iii) tratamento térmico (calcinação) para transformar os cátions maiores em óxidos (pilares), e desta forma fixar a estrutura (DING; KLOPROGGE; FROST, 2001). A Figura 2 ilustra estas etapas. 
Figura 2 - Representação esquemática do processo de pilarização

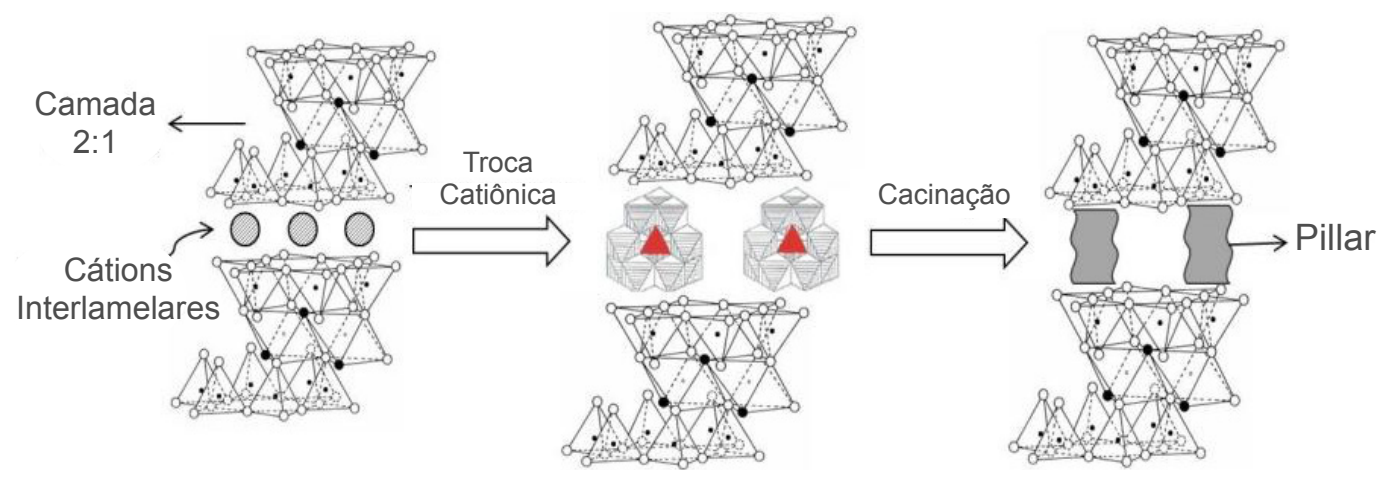

Fonte: Adaptado de BERTELA, 2014.

Um cátion comumente empregado na pilarização de argilas é o íon de Keggin $\left(\mathrm{Al}_{13}=\left[\mathrm{Al}_{13} \mathrm{O}_{4}(\mathrm{OH})_{24} \cdot 12 \mathrm{H}_{2} \mathrm{O}\right]^{+7}\right.$, que além de propiciar o espaçamento entre as camadas, aumentando a área especifica do material e acessibilidade à região interlamelar, possui acidez para levar a cabo reações catalíticas. (TEIXEIRA- NETO; TEIXEIRA-NETO 2009).

Comparados com os seus precursores, esses materiais possuem elevada área específica, poros volumosos e ajustáveis, permitindo melhor acessibilidade, maior acidez devido à inserção de óxidos metálicos e exposição da superfície, aumento na estabilidade térmica, bem como propriedades catalíticas associadas aos metais inseridos e ao poder adsortivo que é característico dos argilominerais (BERGAYA; AUOAD; MANDALIA, 2006).

A literatura para síntese de argilas pilarizadas é muito extensa. A maior parte dos trabalhos usa cátions de alumínio para a síntese dos agentes pilarizantes (BALOYI et al. 2018). As soluções deste complexo são feitas através da adição de uma base a um sal de alumínio até razão molar de $\mathrm{OH} / \mathrm{Al}=2,5$.

Em vista do exposto, neste trabalho se resolveu trabalhar com a preparação de argilas pilarizadas com o tradicional íon de Keggin $\left(\mathrm{Al}_{13}\right)$, estudando a quantidade de água em- pregada em mais de uma etapa do processo com a finalidade de avaliar a influência deste parâmetro no procedimento de pilarização. A quantidade de água empregada num processo é muito importante quando se pretende otimizar o mesmo pensando num escalonamento e uso industrial (BERTELLA; PERGHER, 2017a), além de sínteses mais eco-amigáveis (BERTELLA; PERGHER; 2017b).

Para tal, se estudou a síntese de argilas pilarizadas variando a quantidade de água empregada em três etapas do processo: no primeiro momento foi avaliado o uso de água na etapa de pré-expansão da argila; num segundo momento avaliou-se a quantidade de solução de agente pilarizante necessária por grama de argila e, por fim, se avaliou a necessidade das lavagens para remoção do íon cloreto.

\section{Parte Experimental}

$\mathrm{O}$ argilomineral empregado foi a montmorillonita comercial sigma-aldrich .

\section{Preparo da Solução do Agente Pilarizante (íon de Keggin $\mathrm{Al}_{13}$ ):}

Uma solução de $500 \mathrm{~mL}$ de hidróxido de sódio $(\mathrm{NaOH})$ 0,2 mol/L foi gotejada com 
velocidade de $1 \mathrm{~mL} / \mathrm{min}$ em uma solução de $250 \mathrm{~mL}$ de cloreto de alumínio hexahidratado $\left(\mathrm{AlCl}_{3} \cdot 6 \mathrm{H}_{2} \mathrm{O}\right) 0,2 \mathrm{~mol} / \mathrm{L}$, sob agitação constante e em uma temperatura de $60^{\circ} \mathrm{C}$. A solução resultante com razão molar $\mathrm{OH} / \mathrm{Al}=$ 2, pH entre 3,0 e 4.,0 é mantida sob agitação a $60^{\circ} \mathrm{C}$, por $24 \mathrm{~h} \mathrm{e}$, posteriormente, pode ser armazenada ou empregada no processo de pilarização.

\section{Preparo da Argila Pilarizada}

Inicialmente, a argila é pre-expandida com água. Para tal, $3 \mathrm{~g}$ de argila comercial foi dispersa em $300 \mathrm{~mL}$ de água destilada $(1 \mathrm{~g} / 100 \mathrm{~mL})$ e mantida a agitação por $2 \mathrm{~h}$ em temperatura ambiente.

Posteriormente, a esta suspensão de argila, se adiciona a solução do agente pilarizante $(750 \mathrm{~mL})$ e se mantém por mais $2 \mathrm{~h}$ à temperatura ambiente (processo de troca dos cátions naturais de argila pelos íons de Keggin).

Finalmente, a suspensão é filtrada, o material obtido é lavado abundantemente com água destilada, até teste negativo para cloreto, seco durante $12 \mathrm{~h}$ em uma estufa a $60^{\circ} \mathrm{C}$. Posteriormente, o material é calcinado a $300^{\circ} \mathrm{C}$ por $3 \mathrm{~h}$ em uma mufla (a taxa de aquecimento foi de $2^{\circ} \mathrm{C} / \mathrm{min}$ ).

\section{Parâmetros de síntese Estudados}

Para avaliar a etapa de pré-expansão da argila com água, foi realizado um experimento sem esta etapa, portanto a solução pilarizante preparada foi adicionada diretamente a $3 \mathrm{~g}$ de argila seca e mantida sob agitação por $2 \mathrm{~h}$ $\mathrm{e}$, posteriormente, seguindo os passos para obtenção do produto final.

A quantidade de solução do agente pilarizante foi avaliada usando as seguintes quantidades: $100 \mathrm{~mL} / \mathrm{g} ; 200 \mathrm{~mL} / \mathrm{g} ; 250 \mathrm{~mL} / \mathrm{g}$ (padrão) e $300 \mathrm{~mL} / \mathrm{g}$.

O efeito da lavagem do produto obtido foi avaliado realizando um experimento sem esta etapa de lavagem, sendo, então, o material, após a filtração, seco e em seguida calcinado.

\section{Caracterização dos Materiais}

Os materiais obtidos foram caracterizados por difração de raios X (DRX) empregando um equipamento Bruker D2 Phaser utilizando radiação $\mathrm{CuK} \alpha(\lambda=1,54 \AA)$ com um filtro de $\mathrm{Ni}$, com passo de $0,02^{\circ}$, corrente de $10 \mathrm{~mA}$, voltagem de $30 \mathrm{kV}$, utilizando um detector Lynxeye, com fenda divergente de $0,1 \mathrm{~mm}$, tempo de $0,2 \mathrm{~s}$, anti-air scattering screen de $1 \mathrm{~mm}$ e fenda convergente de $3 \mathrm{~mm}$.

Os espaçamentos basais das amostras foram calculados através da Lei de Bragg ( $\mathrm{n} \lambda$ $=2 \operatorname{dsen} \theta$ ), sendo $\mathrm{n}$ um número inteiro, $\lambda$ é o comprimento de onda da radiação incidente, $\mathrm{d}$ é a distância interplanelar e $\theta$ é o ângulo de incidência.

\section{Resultados e Discussão}

A Figura 3 apresenta o difratograma de raios-X da argila sem tratamentos, onde se observa o pico 001 a $2 \theta$ de $7,2^{\circ}$ característico do argilomineral montmorilonita (MOORE, 1997). Também se observa pequena quantidade de quartzo $\left(2 \theta \sim 26^{\circ}\right)$ que é uma impureza comum em argilas.

Através da posição deste pico 001, se calcula pela lei de Brag o espaçamento basal, obtendo-se o valor de 1,22 $\mathrm{nm}$. Segundo a literatura (CARMO, 2015), quando o cátion interlamelar é $\mathrm{Na}^{+}$, na montmorillonita seca ou hidratada o d(001) está em torno de 1,2 $\mathrm{nm}$ a $1,4 \mathrm{~nm}$. Então confirma-se que se trata de uma argila tipicamente montmorillonita na forma sódica.

Nesta argila após o processo de pilarização, a estrutura lamelar se mantém, entretanto, a distância entre as camadas vão ser maiores, uma vez que agora se tem pilares mantendo-as separadas. Isto se observa pelo 
deslocamento deste pico 001 para ângulos menores indicando um aumento do espaçamento basal para a aproximadamente $1,8 \mathrm{~nm}$ conforme apresentado nas Figuras 4A (não calcinada) e 4B (calcinadas).

Figura 3 - Difratograma de raios-X da Argila Comercial

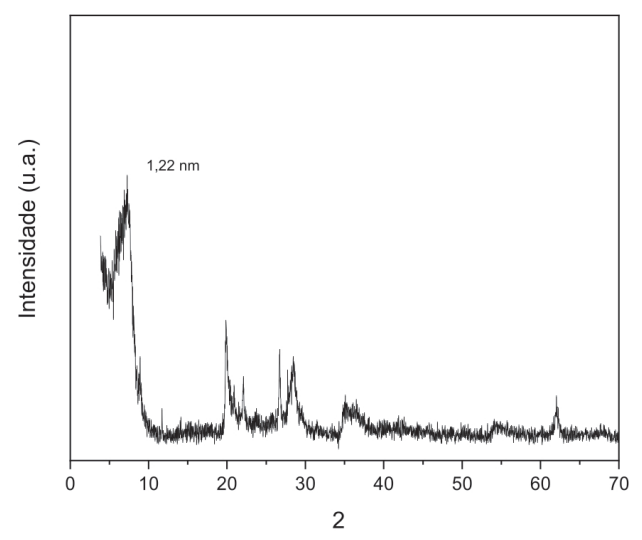

Ao comparar as amostras antes e após calcinação observa-se uma diminuição do espaçamento basal que está de acordo com o reportado na literatura (PERGHER, 1993). Isto ocorre devido a contração do pilar ao ocorrer a transformação do íon de keggin em óxidos de Alumínio (equação 1).

$$
\begin{gathered}
{\left[\mathrm{Al}_{13} \mathrm{O}_{4}(\mathrm{OH})_{24} \cdot 12 \mathrm{H}_{2} \mathrm{O}\right]^{+7} \rightarrow 6,5 \mathrm{Al}_{2} \mathrm{O}_{3}+7} \\
\mathrm{H}^{+}+20,5 \mathrm{H}_{2} \mathrm{O}
\end{gathered}
$$

Ao comparar os matérias obtidos com e sem a pré-expansão, observam-se valores de espaçamento basal um pouco maiores e picos 001 mais intensos para as amostras pré-expandidas, indicando que a pré-expansão favorece uma pilarização mais ordenada e efetiva, mas dependendo das propriedades finais do material que se deseja, ela pode ser retirada ou minimizada.

Outro parâmetro importante é a quantidade de solução pilarizante a ser empregada, pois visando a um aumento de escala da sín- tese desses materiais, esta quantidade deve ser otimizada. Os resultados do estudo deste parâmetro estão apresentados nas Figuras 5A e 5B, representando as amostras antes e após calcinação, respectivamente.

Figura 4 - Difratogramas de raios-X das amostras pré-expandidas (a) e não pré-expandidas (b) em água antes (A) e após calcinação (B)

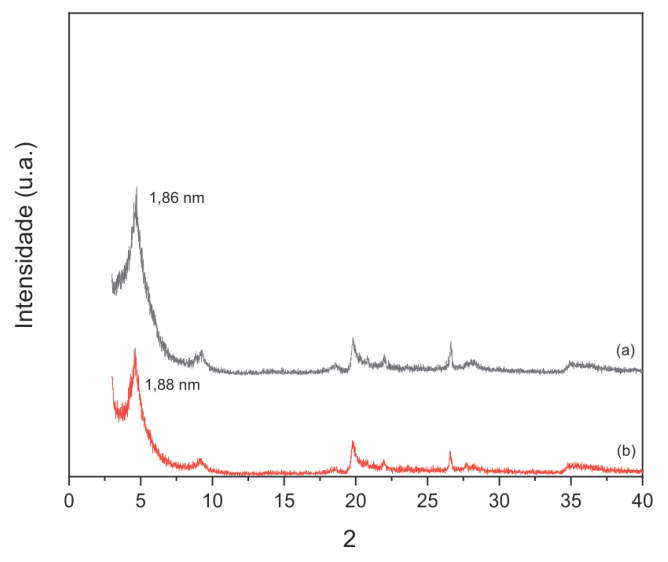

(A)

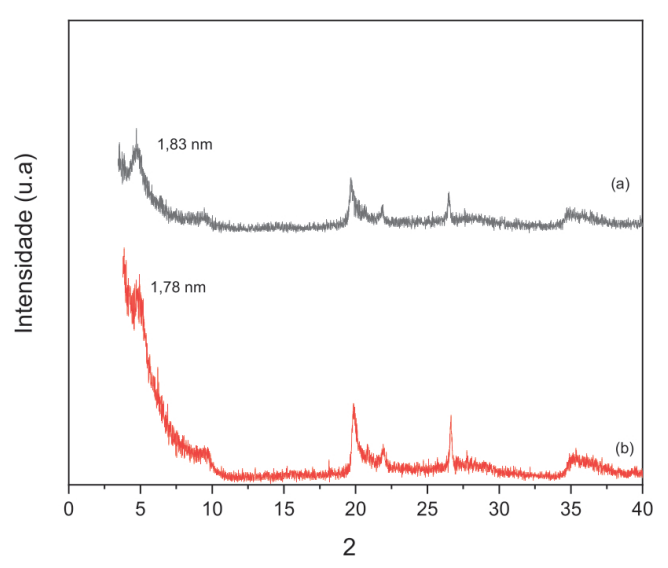

(B)

Os melhores resultados foram obtidos com a quantidade de $100 \mathrm{~m}$ L da solução pilarizate por $\mathrm{g}$ de argila, obtendo valores de espaçamento basal de $1,93 \mathrm{~nm}$ e após calcinação de $1,85 \mathrm{~nm}$, indicando a formação de um material pilarizado com boas propriedades de espaçamento e boa distribuição dos 
pilares, evidenciada pelo pico 001 intenso. Ao aumentar a quantidade da solução pilarizante para $200 \mathrm{~mL} / \mathrm{g}, 250 \mathrm{~mL} / \mathrm{g}$ e $300 \mathrm{~mL} / \mathrm{g}$ ocorre diminuição gradual da intensidade desse pico 001 e do espaçamento basal. Este resultado indica que é possível reduzir a quantidade de agente pilarizante por grama de argila em mais da metade do que se vem utilizando nas sínteses padrões $(250 \mathrm{~mL} / \mathrm{g})$, economizando solução pilarizante e água, no processo. Este efeito pode estar ocorrendo devido ao meio estar mais concentrado em relação à quantidade de argila, favorecendo o contato e, portanto, a pilarização.

Figura 5 - Difratogramas de raios-X das amostras obtidas com diferentes quantidades de solução de agente pilarizante (a) $100 \mathrm{~mL} / \mathrm{g}$; (b) $200 \mathrm{~mL} / \mathrm{g}$; (c) $250 \mathrm{~mL} / \mathrm{g}$; e (d) $300 \mathrm{~mL} / \mathrm{g}$ antes (A) e após (B) calcinação

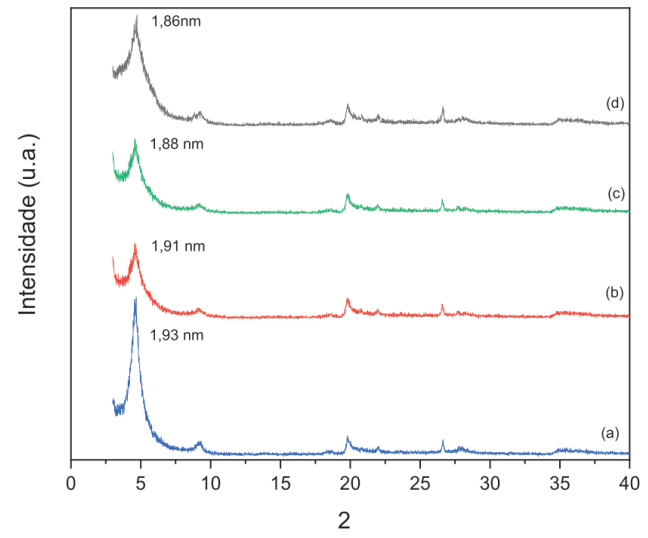

(A)

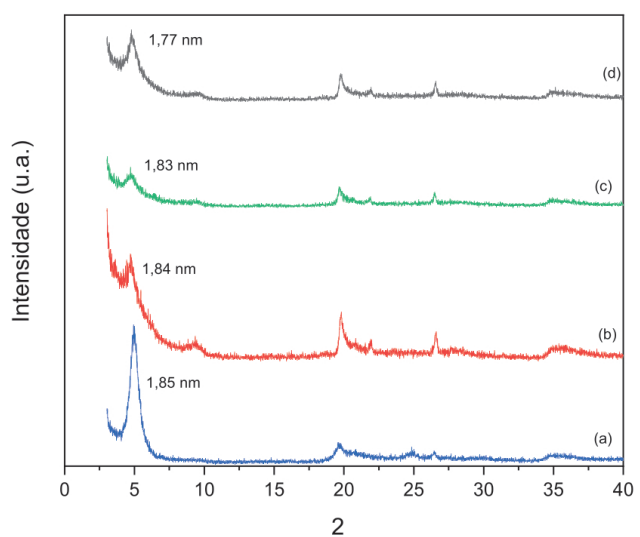

(B)
Figura 6 - Amostras obtidas com (a) e sem (n) etapa de Lavagem, antes (A) e após calcinação (B)

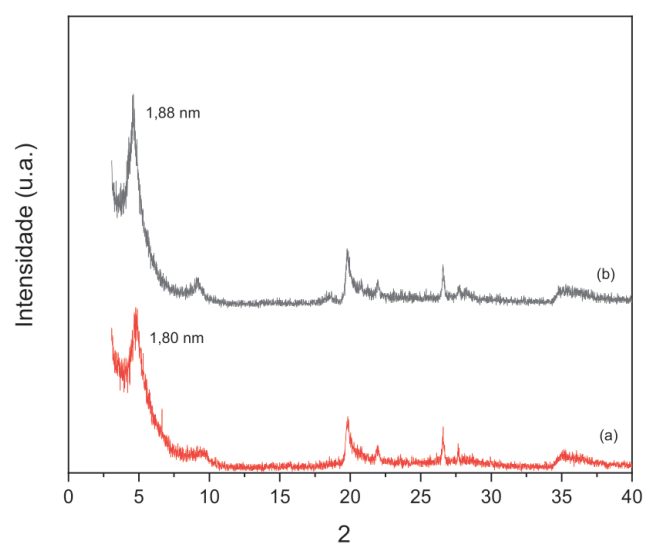

(A)

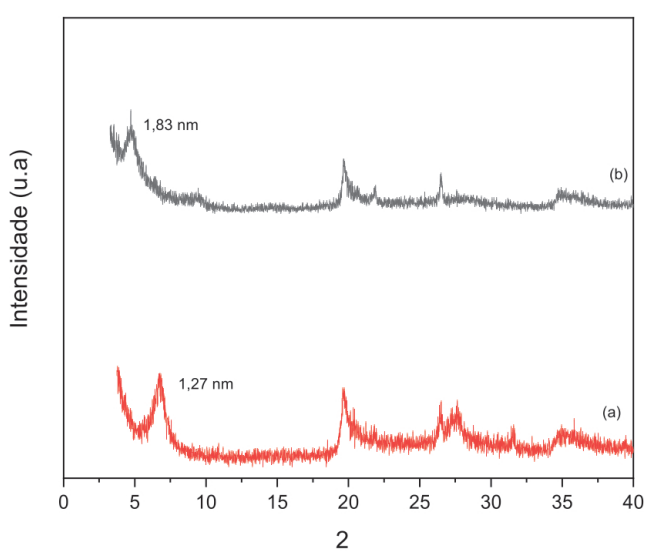

(B)

A Figura 6 apresenta os difratogramas dos materiais obtidos com e sem a etapa de lavagem, antes e depois da calcinação. A etapa de lavagem consiste na passagem de água deionizada na amostra filtrada até a remoção total dos cloretos (observado através de teste de presença de cloretos na água de lavagem empregando uma solução de $\mathrm{AgNO}_{3}$ ). Observa-se que nas amostras antes da calcinação, a lavagem não apresenta diferença tão significativa quanto à expansão, seja nos valores de espaçamento basal, seja na intensidade da reflexão do pico em (001). Porém, quando analisamos as amostras calcinadas, percebemos a diminuição drástica 
nos valores do espaçamento basal, indicando a destruição do material pilarizado. Esse resultado, reflete a importância do processo de lavagem, pois íons cloretos remanescentes destruirão a estrutura durante o processo de calcinação, além de contribuir para uma distribuição igual de pilares entre as camadas, formando espaçamentos intercamadas maiores, afetando o tamanho dos poros e sua uniformidade (SALERNO et al., 2001).

\section{Conclusão}

Três parâmetros na síntese de argilas pilarizadas que envolvem o uso de água foram avaliados no sentido de minimizar este componente visando a otimização do processo de síntese para futuro escalonamento. Estes parâmetros foram: a pré-expansão da argila em água; a quantidade de solução de agente pilarizante; e a etapa de lavagem. Como res- posta, se observou a formação dos materiais pilarizados e seu espaçamento basal obtidos pela difração de raios $-X$.

A pré-expansão da argila com água mostrou um leve aumento no espaçamento basal, e uma melhor organização no material pilarizado. Entretanto, pode ser uma etapa a ser dispensável, uma vez que na etapa seguinte de adição da solução do agente pilarizante a mesma fará a expansão das lamelas e pilarização do material.

A quantidade da solução pilarizante necessária pode ser reduzida. Observou-se ser necessário somente $100 \mathrm{~mL} / \mathrm{g}$ em vez dos $250 \mathrm{~mL} / \mathrm{g}$ normalmente empregados uma redução bem significativa quando se pensa no escalado das sínteses.

A etapa de Lavagem até eliminação de cloretos mostrou ser uma etapa fundamental para manter as amostras pilarizadas após a calcinação das mesmas.

\section{REFERÊNCIAS}

AZEEZ, A. A.; RHEE, K. Y.; PARK, S. J.; HUI, D. Epoxy clay nanocomposites - Processing, properties and applications: A review. Composites Part. B: Engineering, v. 45, n. 1, p. 308-320, 2013.

BALOYI, J.; NTHO, T.; MOMA, J. Synthesis and application of pillared clay heterogeneous catalysis for wastewater treatment: a review. RSC Advances, v. 8, p. 5197-5211, 2018.

BERGAYA, F.; AOUAD, A.; MANDALIA, T. Pillared Clays and Clay Minerals. Capítulo 7.5, Handbook of Clay Science. Elsevier, 2006.

BERTELLA, F. Síntese e caracterização de argilas pilarizadas com pilares mistos Al/Co.

Dissertação. (Mestrado em Química) UFRN, Natal, RN, 2014.

BERTELLA, F.; PERGHER, S. Scale Up Pillaring: A study of the parameters that influence the process. Materials, v. 10, n. 7, p. 712, 2017a.

BERTELLA, F.; PERGHER, Reuse of Pillaring Agent in Sequential Bentonite Pillaring Processess. Materials, v. 10, n. 7, p. 705, 2017 b.

CARDONA, Y.; KORILI, S.A.; GIL, A. Understanding the formation of $\mathrm{Al}_{13}$ and $\mathrm{Al}_{30}$ polycations to the development of microporous materials based no Al13- and Al130-PILC montmorillonite: a review. Applied Clay Science, v. 203, p.105996, 2021. 
CARMO, A. L. V. do. Avaliação da influência dos cátions $\mathrm{Na}^{+}, \mathrm{Ca}^{2+}$ e $\mathrm{Mg}^{2+}$ na desativação de Ca, Mg-bentonitas. 2015. 70 f. Dissertação (Mestrado em Geologia e Geoquímica) UFPA, Belém, PA, 2015.

COGO, J. M. Caracterização e funcionalização de argila esmectita de alteração basáltica e utilização na remoção de corante com processo de adsorção: estudo da cinética e equilíbrio do processo. 2011. 99 f. Dissertação (Mestrado em Geociências) - UFMT, Cuiabá, MT, 2011.

DING, Z.; KLOPROGGE, J. T.; FROST, R. L. Porous Clays and Pillared Clays- Based Catalysts. Part 2: A Review of the Catalytic and Molecular Sieve Applications. Journal of Porous Materials, v.8, p. 273-293, 2001.

GUGGENHEIM, S; ADAMS, J. M.; BAIN, D. C.; BERGAYA, F.; BRIGATTI, M. F.; DRITS, V. A.; FORMOSO, M. L. L.; GALÁN, E.; KOGURE, T. e STANJEK, H. Summary of recommendations of nomenclature committes relevant to clay mineralogy: repot of Association Internationale Pour L'etude des Argiles (AIPEA) nomenclature committee for 2006. Clays and Clay Minerals, v. 54, n. 6, p. 761-772, 2006.

MOORE, D. M.; R. C.; REYNOLDS, Jr. X-ray diffraction and the identification and analysis of clay minerals. 2nd ed. Oxford University Press, Oxford, 1997.

ODOM I. E. Smectites clay minerals: properties and uses. Philosophical Transactions of Royal Society of London A, v. 311, p. 391-409, 1984.

PERGHER, S. B .C. Preparação e Propriedades de uma Argila Paranaense Pilarizada com Polihidroxications de Aluminio, 1993. Dissertação. (Mestrado em Engenharia Química) Universidade Estadual de Maringá, Brasil, 1993.

PERGHER, S. B. C.; SPRUNG, R. Pilarização de uma brasileira com poliidróxicátions de alumínio: preparação, caracterização e propriedades catalíticas. Química Nova, v. 28, n. 5, p. 777-782, 2005.

ROMANZINI, D. Efeito da organossilanização de argilas montmorilonita (Mt) nas propriedades de nanocompósitos Mt/poliéster e Mt/fibra de vidro moldados por RTM. 2016. 123f. Tese (Doutorado em Engenharia) - UFRGS, Porto Alegre, RS, 2016.

SALERNO, P.; ASENJO, M. B.; MENDIOROZ, S. Influence of preparation method on thermal stability and acidity of Al-PILCs. Thermochimica Acta, v. 379, n. 1-2, p. 101-109, 2001.

TEIXEIRA-NETO, E.; Teixeira-NETO. A. A. Modificação química de argilas: desafios científicos e tecnológicos para obtenção de novos produtos com maior valor agregado Química Nova, v. 32, n. 3, p. 809-817, 2009.

WEN, K.; ZHU, J.; CHEN, H.; MA,L.; LIU, H; ZHU, R.; XI, Y. and HE, H. Arrangement models of kegging - Al30 and kegging Al13 in the interlayer of Montmorillonite and the impacts of pillaring on surface acidity: A comparative study on Cattalytic Oxidation of Toluene. Langmuir, v. 35, p. 382-290, 2019. 
